Original Article

\title{
Hepatoprotective effect of sodium hydrosulfide on hepatic encephalopathy in rats
}

\author{
Kyoung Wan Kwon", Yoonjin Nam\#, Won Seok Choi", Tae Wook Kim, Geon Min Kim, and Uy Dong Sohn* \\ Department of Pharmacology, College of Pharmacy, Chung-Ang University, Seoul 06974, Korea
}

\author{
ARTICLE INFO \\ Received February 4, 2019 \\ Revised May 17, 2019 \\ Accepted June 3, 2019 \\ *Correspondence \\ Uy Dong Sohn \\ E-mail: udsohn@cau.ac.kr
}

\section{Key Words}

Hepatic encephalopathy

Hydrogen sulfide

Inflammation

\#These authors contributed equally to this work.

\begin{abstract}
Hydrogen sulfide is well-known to exhibit anti-inflammatory and cytoprotective activities, and also has protective effects in the liver. This study aimed to examine the protective effect of hydrogen sulfide in rats with hepatic encephalopathy, which was induced by mild bile duct ligation. In this rat model, bile ducts were mildly ligated for 26 days. Rats were treated for the final 5 days with sodium hydrosulfide (NaHS). NaHS ( $25 \mu \mathrm{mol} / \mathrm{kg}), 0.5 \%$ sodium carboxymethyl cellulose, or silymarin $(100 \mathrm{mg} / \mathrm{kg})$ was administered intraperitoneally once per day for 5 consecutive days. Mild bile duct ligation caused hepatotoxicity and inflammation in rats. Intraperitoneal NaHS administration reduced levels of aspartate aminotransferase and alanine aminotransferase, which are indicators of liver disease, compared to levels in the control mild bile duct ligation group. Levels of ammonia, a major causative factor of hepatic encephalopathy, were also significantly decreased. Malondialdehyde, myeloperoxidase, catalase, and tumor necrosis factor- $\alpha$ levels were measured to confirm antioxidative and anti-inflammatory effects. N-Methyl-D-aspartic acid (NMDA) receptors with neurotoxic activity were assessed for subunit NMDA receptor subtype 2B. Based on these data, NaHS is suggested to exhibit hepatoprotective effects and guard against neurotoxicity through antioxidant and anti-inflammatory actions.
\end{abstract}

\section{INTRODUCTION}

Hepatic encephalopathy (HE) is caused by acute or chronic liver disease, or severe portosystemic venous shunting, which results in severe hepatocellular dysfunction and the production of various toxic substances that affect brain tissue and cause abnormal neurotransmission [1]. The symptoms of HE include unconsciousness, behavioral and personality changes, and neuropsychiatric syndromes that can cause neurological abnormalities. Initial symptoms may include helplessness, restlessness, and irritability. In severe cases, unconscious turbidity, ataxia, bradykinesia, and coma may occur. It is thought that HE occurs through the actions of nitrogen compounds derived from the intestines, particularly ammonia, that act as neurotoxic substances; recently, various compounds have been proposed to act together [2-4].
HE can be classified into three types: A, B, and C [5]. Type A $\mathrm{HE}$ is associated with acute liver failure, hepatic devascularization, hepatectomy, galactosamine, and acetaminophen, among other effects, which can be used to prepare animal models of type A HE. Type B HE is associated with the portal vein branch in the absence of hepatic liver disease, and portacaval anastomosis and grafted portal vein stenosis can be used to prepare animal models of type B HE. Type C HE is associated with cirrhosis and portal hypertension or portosystemic shunts; bile duct ligation (BDL) can be used to prepare animal models of type C HE. Cirrhosis can be induced in many ways, but BDL is often used to prepare reproducible models because it is difficult to produce consistent lesions in each animal [6]. BDL can cause liver failure, jaundice, cirrhosis, portal hypertension, and hyperammonemia, among other effects [7-9]. Liver damage models induced by BDL are (i) \$ This is an Open Access article distributed under the terms of the Creative Commons Attribution Non-Commercial License, which permits unrestricted non-commercial use, distribution, and reproduction in any medium, provided the original work is properly cited. Copyright @ Korean J Physiol Pharmacol, pISSN 1226-4512, eISSN 2093-3827
Author contributions: U.D.S. organized and supervised all procedures. K.W.K., Y.N., and T.W.K. performed data analysis and revised the work protocol and manuscript. W.S.C. and G.M.K. participated in research design and contributed analytic techniques and tools. 
widely used [10], which are also used to induce HE. When the bile ducts are ligated by BDL, the liver is injured by induction of inflammation, causing fibrosis [11]. Blood ammonia levels rise due to liver damage and liver inflammation, and the resulting hyperammonemia is the cause of hepatic encephalopathy [12].

However, BDL cannot be used as a long-term experimental animal model because death occurs within 5-6 weeks and causes unnecessary suffering. However, mild BDL (MBDL) can induce hepatic coma with fewer effects on rats compared to BDL, as only 3 of 5 biliary ducts are excised [13].

N-Methyl-D-aspartic acid (NMDA) receptors are learning and memory-related receptors that are primarily found in nerve cells [14]. Therefore, NMDA receptors are an important index of HE or brain damage [15]. High ammonia levels in HE activate NMDA receptors and increase their expression. In acute hyperammonemia, excessive activation of the increased NMDA receptors causes nerve damage and death [16]. In chronic hyperammonemia and liver failure, however, ammonia levels are not high enough to cause death, and model animals can use other pathways to adapt to activation of the NMDA receptor-associated signaling pathways, such as the glutamate-nitric oxide (NO)-cGMP pathway. These adaptive responses help to protect against neurotoxicity caused by excessive activation of NMDA receptors, but impair the normal physiology of neurons and contribute to neurological disorders, such as cognitive and memory disorders [17,18]. NMDA receptors consist of an NR1 subunit and NR2A-D. NR2B plays an important role in the hippocampus and is related to memory and learning $[19,20]$.

Hydrogen sulfide $\left(\mathrm{H}_{2} \mathrm{~S}\right)$ is a gaseous signaling molecule generated in mammalian cells. At high concentrations, $\mathrm{H}_{2} \mathrm{~S}$ acts as a toxic agent by inhibiting cytochrome $c$ oxidase and stopping ATP production, while at low concentrations, $\mathrm{H}_{2} \mathrm{~S}$ represents a third most common inorganic gaseous mediator, following $\mathrm{NO}$ and carbon monoxide [21-23]. $\mathrm{H}_{2} \mathrm{~S}$ exerts protective effects by reducing inflammatory substances, showing antioxidant activities, and inhibiting cell apoptosis in the liver [24,25]. In addition, $\mathrm{H}_{2} \mathrm{~S}$ protects the liver from hepatic ischemia/reperfusion (HIR) injury and increases subunit NR2B expression of NMDA receptors in HIR-induced cognitive dysfunction $[26,27]$. However, excessive activation of NMDA receptors is a problem in $\mathrm{HE}$; if $\mathrm{H}_{2} \mathrm{~S}$ increases NMDA receptor levels, neurotoxic effects may occur.

This study aimed to identify liver-protective effects through antioxidant and anti-inflammatory activities and to determine whether the effects of hydrogen sulfide lead to neurotoxic protective effects in hepatic coma through the NR2B subunit.

\section{METHODS}

\section{Animals}

Male Sprague-Dawley (SD) rats, weighing 250-300 g, were purchased from Samtako (Osan, Korea). Rats were housed at room temperature $\left(24^{\circ} \mathrm{C}-25^{\circ} \mathrm{C}\right)$ and exposed to a 12/12-h lightdark cycle. Water and food were provided ad libitum. Experiments were approved by performed in accordance with guidelines of the Institutional Animal Care Use Committee of Chung-Ang University (IACUC 2017-00076).

\section{Drugs}

Sodium hydrosulfide hydrate and silymarin were purchased from Sigma-Aldrich (St. Louis, MO, USA). All solutions were freshly prepared on the day of the experiment.

\section{MBDL model and experimental design}

Rats with hepatic encephalopathy were induced by MBDL [13]. The operation was performed under isoflurane anesthesia. A midline abdominal incision was made along the linea alba and ligation was performed at the site where three of the five biliary ducts were joined (Supplementary Fig. 1). The sham group was treated using the same procedure except for ligation. After a three-week hepatic encephalopathy induction period, the rats subjected to MBDL surgery received intraperitoneal administration of NaHS $(25 \mu \mathrm{mol} / \mathrm{kg})$ for 5 consecutive days. The control, sham, and MBDL groups received intraperitoneal administration of an equal volume of $0.5 \%$ sodium carboxymethyl cellulose. The silymarin group, used as a positive control, received intraperitoneal administration of silymarin $(100 \mathrm{mg} / \mathrm{kg})$. At $24 \mathrm{~h}$ after the fifth administration of drug, rats were sacrificed by diethyl ether; blood, liver, and brain were collected and prepared for further assays ( $\mathrm{n}=6$ for all groups).

\section{Preparation and biochemical assay of serum samples}

After sacrifice of the rats, whole blood was collected from the inferior vena cava using a vacutainer (BD Biosciences, San Jose, CA, USA). Collected blood samples were centrifuged at 10,000 $\times g$ for $20 \mathrm{~min}$ at $4^{\circ} \mathrm{C}$. Serum was collected and stored at $-80^{\circ} \mathrm{C}$ until subsequent use.

\section{Hematoxylin and eosin (H\&E) staining}

Liver tissues were dissected and fixed in 10\% formalin solution. Liver samples were dehydrated with alcohol, cleared with xylene, and then infiltrated and embedded with paraffin. Paraffin blocks were cut to $5 \mu \mathrm{m}$-thick sections using a microtome and stained with H\&E. Stained tissues were observed with a Leica DMR 6000 microscope (Wetzlar, Germany).

\section{Measurement of serum parameters}

Alanine aminotransferase (ALT), aspartate aminotransferase 
(AST), myeloperoxidase (MPO), and catalase (CAT) activities and the malondialdehyde (MDA) concentration were measured using an ALT activity assay kit, AST activity assay kit, MPO activity assay kit, catalase assay kit, and lipid peroxidation (MDA) assay kit (Biovision, Milpitas, CA, USA). Ammonia concentration was measured using an ammonia assay kit (Abcam, Cambridge, UK).

\section{Western blotting assay}

Each section of brain tissue was lysed in radio immunoprecipitation assay buffer containing phosphatase inhibitor cocktail and a protease inhibitor mixture. Supernatants were obtained by centrifugation and stored at $-80^{\circ} \mathrm{C}$ until subsequent use in western blotting. Next, $25 \mu \mathrm{g}$ protein in each sample was separated by $7.5 \%$ SDS-PAGE and transferred to a nitrocellulose (NC) membrane. The NC membrane was blocked with $3 \%$ bovine serum albumin for $1 \mathrm{~h}$ at room temperature and washed three times for $10 \mathrm{~min}$ per wash with TBS buffer. The NC membrane was incubated at $4^{\circ} \mathrm{C}$ for $24 \mathrm{~h}$ with polyclonal rabbit anti-NR2B antibody $(1: 1,000$; Cell Signaling, Danvers, MA, USA), polyclonal mouse anti-tumor necrosis factor (TNF)- $\alpha$ antibody (1:250; Santa Cruz Biotechnology, Dallas, TX, USA), or anti- $\beta$-actin antibody (1:5,000; Santa Cruz Biotechnology), and then washed three times with tris buffered saline with tween buffer for $10 \mathrm{~min}$. The NC membrane was reacted with goat anti-rabbit secondary antibody (1:5,000; Bethyl Laboratories, Inc., Montgomery, TX, USA) or goat anti-mouse secondary antibody (1:5,000; Bethyl Laboratories, Inc.) for $1 \mathrm{~h}$ at room temperature. Bound antibody was detected by electrochemiluminescence agents. Bands were analyzed using the ChemiDoc XRS and quantified using Quantity One software (Bio-Rad, Hercules, CA, USA).

\section{Statistical analysis}

Data are expressed as means \pm standard error of the mean. Statistical comparisons between each experimental group of all data were analyzed by Student's t-test. Differences were considered significant at error probabilities of less than 0.05 .

\section{RESULTS}

\section{Effects of sodium hydrosulfide (NaHS) on liver dysfunction}

ALT and AST are commonly measured biomarkers for liver dysfunction. In this experiment, liver injury was measured by biochemical analysis and histopathological experiments. MBDL surgery significantly increased serum ALT and AST levels compared to those found in the sham control group (Fig. 1A, B). Additionally, NaHS administration significantly reduced serum AST and ALT levels to amounts similar to those observed in the silymarin group, which served as a positive control.

$\mathrm{H} \& \mathrm{E}$ staining was performed to evaluate histopathological changes in tissues. The sham operation group showed no histopathological changes (Fig. 2A). However, MBDL surgery was characterized by inflammatory cell infiltration, hemorrhagic changes, and focal necrosis (Fig. 2B). NaHS and silymarin administration attenuated these histopathological changes (Fig. 2C, D).

\section{Effect of NaHS on ammonia levels in serum}

Ammonia is a major factor causing $\mathrm{HE}$, and its concentration is increased by liver damage. MBDL surgery significantly increased serum ammonia levels (Fig. 3), while NaHS administration significantly reduced serum ammonia levels to values similar to those observed in the silymarin group.

A

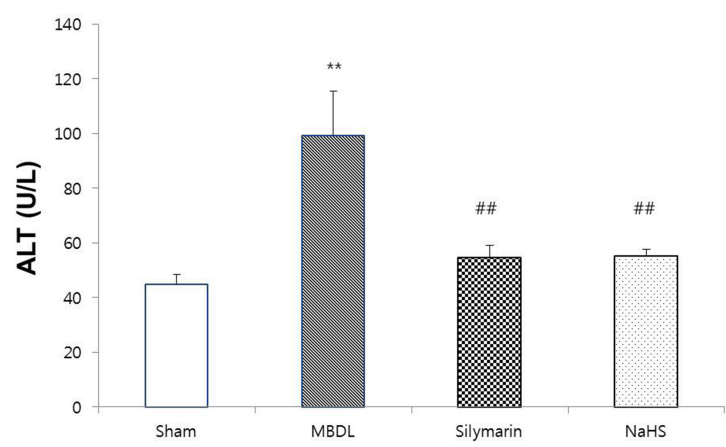

B

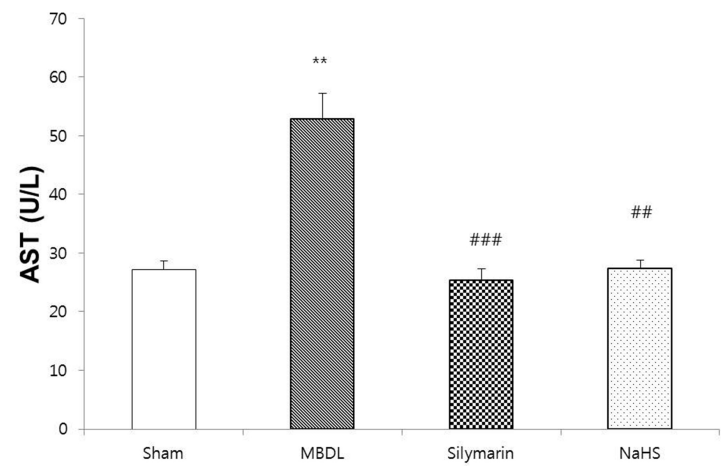

Fig. 1. Effect of sodium hydrosulfide (NaHS) on serum alanine aminotransferase (ALT) and aspartate aminotransferase (AST). (A, B) Mild bile duct ligation (MBDL) surgery increased ALT and AST levels compared to those in the sham control group. NaHS administration significantly decreased serum ALT and AST levels, which were reduced to levels similar to those of the silymarin group. Sham: sham operation control rats, MBDL: MBDL rats, Silymarin: MBDL rats treated with silymarin $(100 \mathrm{mg} / \mathrm{kg})$, NaHS: MBDL rats treated with NaHS (25 $\mu \mathrm{mol} / \mathrm{kg})$. Data are expressed as means \pm standard error of the mean $(n=6) ; * * p$ $<0.01$ compared to sham control, ${ }^{\# \#} p<0.01$ and ${ }^{\# \#} \mathrm{p}<0.001$ compared to MBDL. 

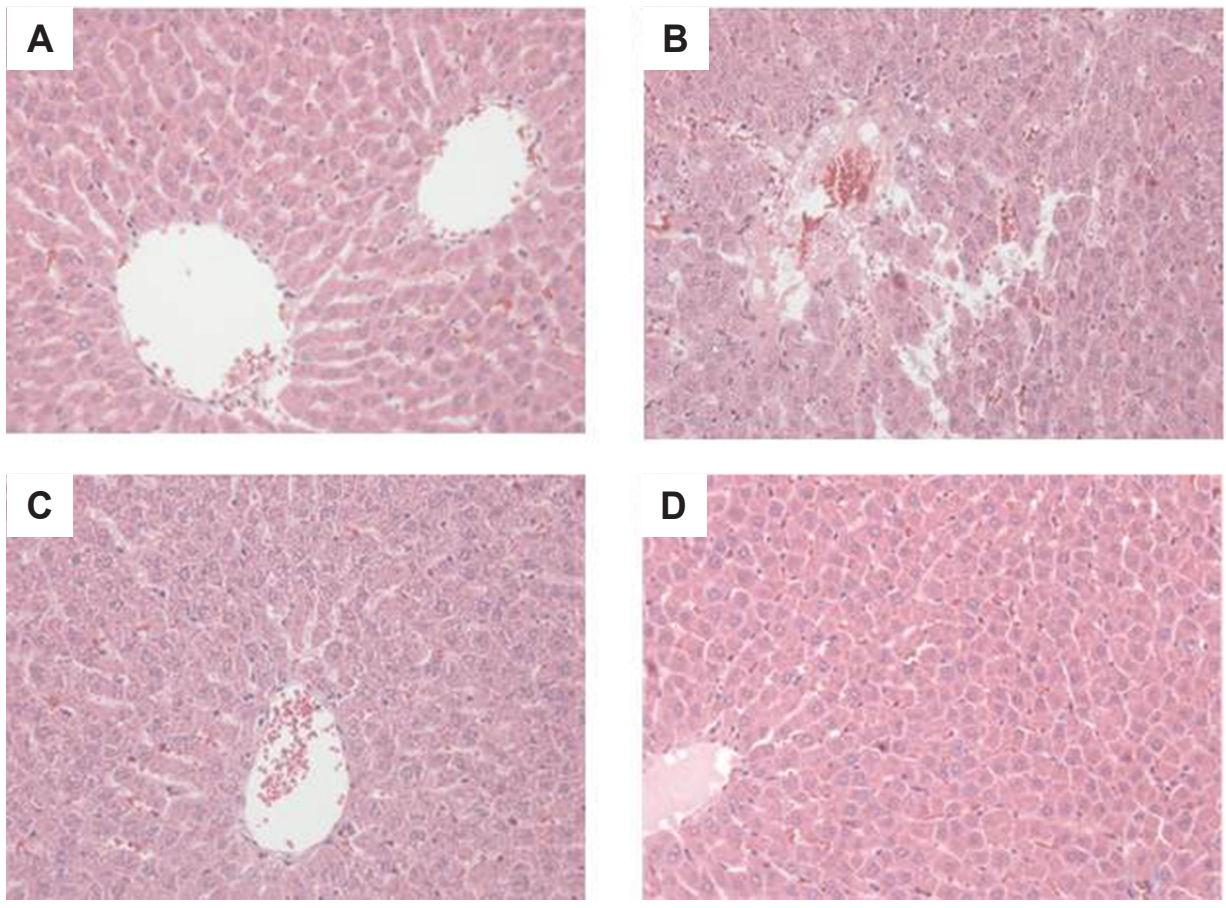

Fig. 2. Photomicrographs of liver sections stained with H\&E. Stained liver sections from the (A) sham-operation group, (B) MBDL group, (C) silymarin (100 $\mathrm{mg} / \mathrm{kg}$ ) treatment group, and (D) NaHS $(25 \mu \mathrm{mol} / \mathrm{kg})$ treatment group. Representative images of each section were captured at $20 \times 10$ magnification. MBDL, mild bile duct ligation; NaHS, sodium hydrosulfide.

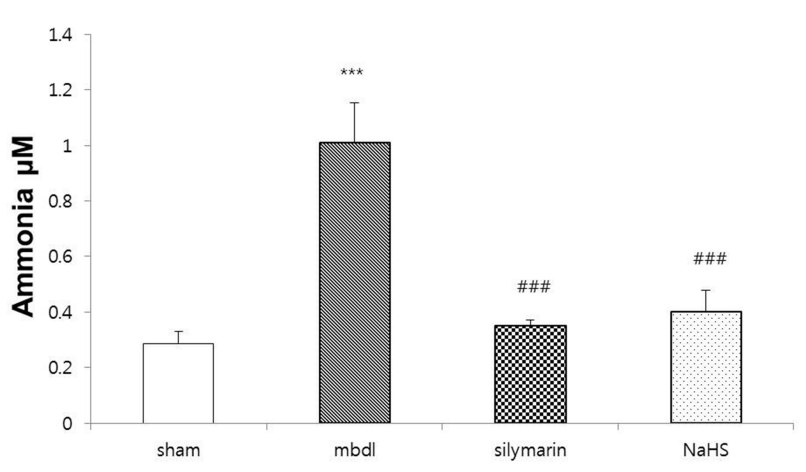

Fig. 3. Effects of sodium hydrosulfide (NaHS) on serum ammonia levels. Animals that underwent mild bile duct ligation (MBDL) surgery showed increased ammonia levels compared to those in the sham control group. NaHS administration significantly decreased ammonia levels to values similar to those observed in the silymarin group. Sham: sham operation control rats, MBDL: MBDL rats, silymarin: MBDL rats treated with silymarin $(100 \mathrm{mg} / \mathrm{kg})$, NaHS: MBDL rats treated with NaHS (25 $\mu \mathrm{mol} / \mathrm{kg}$ ). Data are expressed as means \pm standard error of the mean ( $\mathrm{n}$ $=6$ ); ${ }^{* * *} \mathrm{p}<0.001$ compared to sham control, ${ }^{\# \#} \mathrm{p}<0.001$ compared to MBDL.

\section{Effect of NaHS on serum markers of oxidative stress}

Oxidative stress was measured by biochemical analysis. The MDA concentrations and CAT levels were measured in the serum (Fig. 4A, B). CAT is an antioxidant enzyme that inhibits oxidative stress and protects against liver damage. MBDL surgery significantly increased CAT levels compared to those observed in the sham control group. NaHS administration significantly reduced CAT levels to values similar to those observed in the silymarin group.
MDA is a product of lipid peroxidation. Concentrations of MDA were significantly increased by MBDL surgery compared to those measured in the sham control group. Notably, NaHS administration significantly decreased the MDA concentrations.

\section{Effect of NaHS on liver inflammation}

MPO is a peroxidase enzyme that is abundant in neutrophils and can be used as an indicator of neutrophil infiltration. MPO activities were measured in the serum (Fig. 5A). MBDL surgery significantly increased MPO activity compared to that observed in the sham control group, whereas NaHS administration significantly reduced MPO activity to values similar to those found in the silymarin group.

The cytokine TNF- $\alpha$ is a cell signaling protein that is involved in systemic inflammation. TNF- $\alpha$ levels were measured by western blotting of liver tissues; for quantification, levels were normalized to values of $\beta$-actin staining (Fig. $5 \mathrm{~B}$ ). We found that MBDL surgery increased the levels of liver TNF- $\alpha$. Administration of NaHS reduced levels of TNF- $\alpha$, which had been elevated by MBDL surgery.

\section{Effect of NaHS on NR2B in the hippocampus}

To investigate the effect of MBDL surgery and NaHS on protein expression levels of NR2B, a subunit of the NMDA receptor, hippocampus tissues were harvested and western blotting was performed (Fig. 6). MBDL surgery significantly increased the expression levels of NR2B protein in the hippocampus compared to those observed in the sham group. In contrast, administration of NaHS significantly reduced the expression levels of NR2B protein 
A

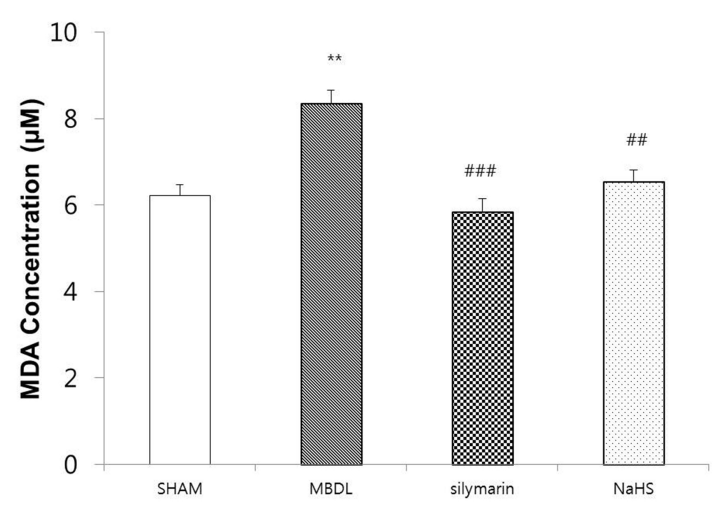

B

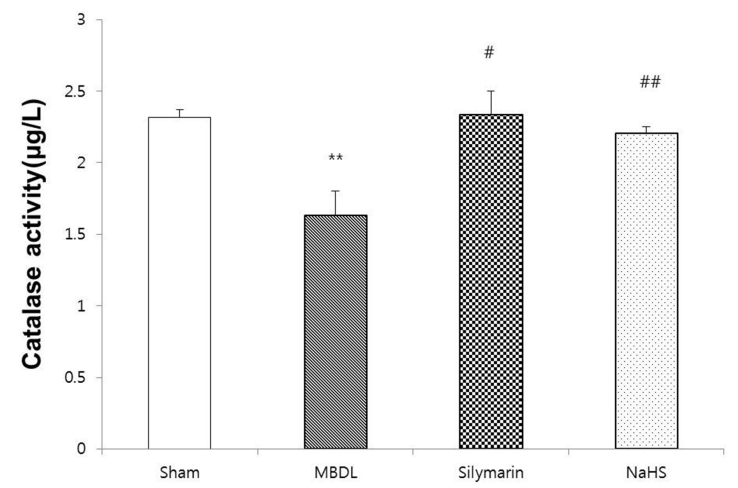

Fig. 4. Effect of sodium hydrosulfide (NaHS) on serum malondialdehyde (MDA) and catalase (CAT) levels. (A) Animals that underwent mild bile duct ligation (MBDL) surgery showed increased MDA levels compared to those observed in the sham control group. NaHS administration significantly decreased MDA levels to values similar to those in the silymarin group. (B) MBDL surgery significantly reduced CAT levels compared to those observed in the sham control group. NaHS administration significantly increased CAT levels to those similar to those found in the silymarin group. Sham: sham operation control rats; MBDL: MBDL rats, silymarin: MBDL rats treated with silymarin $(100 \mathrm{mg} / \mathrm{kg})$, NaHS: MBDL rats treated with NaHS $(25 \mu \mathrm{mol} / \mathrm{kg})$. Data are expressed as means \pm standard error of the mean $(n=6) ; * * p<0.01$ compared to sham control, ${ }^{\#} p<0.05,{ }^{\# \#} p<0.01$ and ${ }^{\# \# \#} p<0.001$ compared to MBDL.

in the hippocampus.

\section{DISCUSSION}

NaHS is known to exert anti-inflammatory and antioxidant effects at low concentrations and has also been found to exhibit hepatoprotective effects $[24,25]$. In this study, the effects of hydrogen sulfide on MBDL-induced type 3 HE were investigated.

In addition, $\mathrm{H}_{2} \mathrm{~S}$ showed a protective effect on cognitive dysfunction induced by HIR by increasing the expression level of the NR2B receptor, which is an important factor in memory and learning processes [27]. However, in HE, NR2B receptor levels are high, which causes side effects [28]. In this study, the effects of $\mathrm{H}_{2} \mathrm{~S}$ on NR2B subunit levels that were increased by HE were investigated.
A

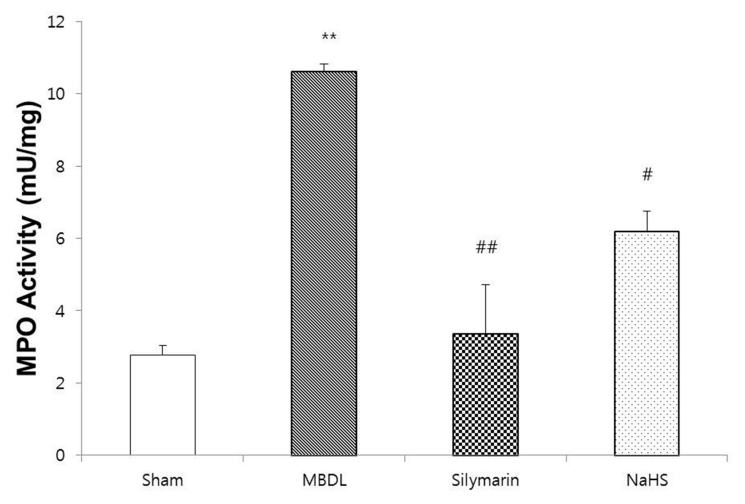

B

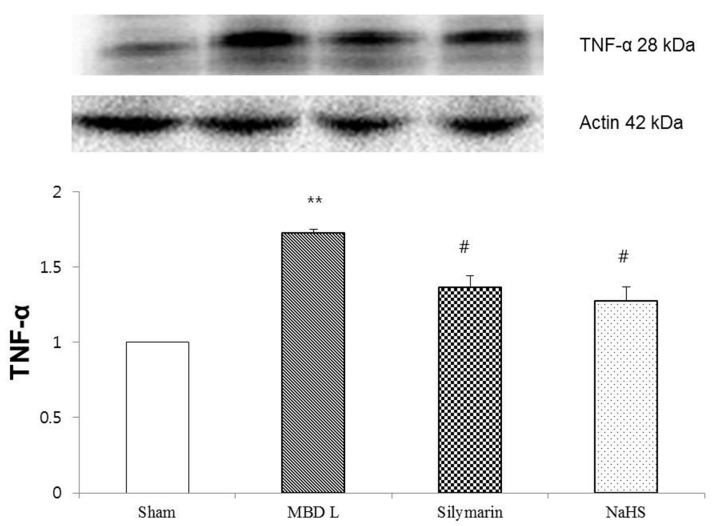

Fig. 5. Effects of sodium hydrosulfide (NaHS) on serum myeloperoxidase (MPO) and liver tumor necrosis factor (TNF)- $\alpha$ levels. (A) Mild bile duct ligation (MBDL) surgery significantly increased MPO activity compared to that observed in the sham control group. NaHS administration significantly decreased MPO activity. (B) MBDL surgery also increased TNF- $\alpha$ expression compared to that observed in the sham control group. NaHS administration significantly decreased serum TNF- $\alpha$ expression to levels similar to those found in the silymarin group. Sham: sham operation control rats, MBDL: MBDL rats, silymarin: MBDL rats treated with silymarin $(100 \mathrm{mg} / \mathrm{kg})$, NaHS: MBDL rats treated with NaHS $(25 \mu \mathrm{mol} / \mathrm{kg})$. Data are expressed as means \pm standard error of the mean $(n=6) ; * * p<0.01$ compared to sham control, ${ }^{*} p<0.05$ and $\# \mathrm{p}<0.01$ compared to MBDL.

If the bile duct is ligated by BDL, bile acid that has not been released will cause serious damage to the liver [7-9]. Stagnant hydrophobic bile acids can damage the mitochondrial electron transport system and produce ROS in the liver. ROS cause an inflammatory response and alter the equilibrium between antioxidant and pro-oxidant activities [29-31]. The damaged liver fails to metabolize ammonia properly and accumulates ammoniainduced type $3 \mathrm{HE}[6]$.

In this study, MBDL surgery elevated liver dysfunction indicators, such as ALT and AST. By contrast, administration of NaHS restored liver dysfunction. Analysis of $\mathrm{H} \& \mathrm{E}$ stained of liver tissue also supported these findings.

It was well known that silymarin exerts protective effects with regard to liver damage. It ameliorated liver damage by reducing lipid peroxidation and free radical activity, protecting hepatocyte 


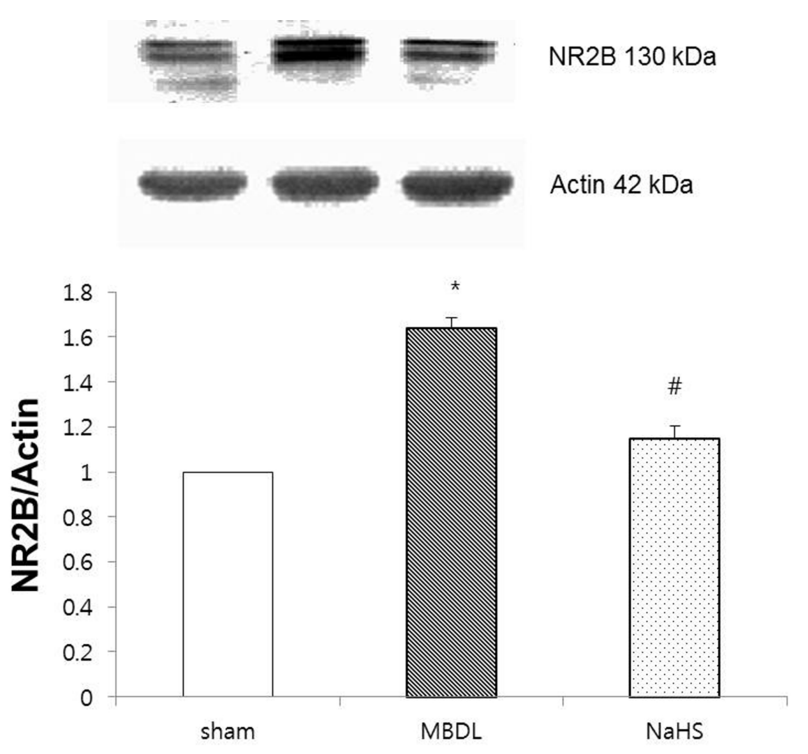

Fig. 6. Effect of sodium hydrosulfide (NaHS) on hippocampus NR2B protein levels. MBDL surgery increased the expression of N-Methyl-Daspartic acid receptor subtype $2 B$ (NR2B) protein in the hippocampus compared to that observed in the sham group. Administration of $\mathrm{NaHS}$ significantly reduced the expression of NR2B protein in the hippocampus. Sham: sham operation control rats, MBDL: MBDL rats, NaHS: MBDL rats treated with NaHS $(25 \mu \mathrm{mol} / \mathrm{kg})$. Data are expressed as means \pm standard error of the mean $(n=6) ;{ }^{*} p<0.05$ compared to sham control, \# $\mathrm{p}<0.05$ compared to MBDL.

membranes, and promoting liver cell regeneration [32]. This study used silymarin as a positive control for a liver damage index.

Ammonia is considered to be the most important causative agent of HE. Ammonia that enters the liver through the portal vein is normally converted to urea and glutamine through the urea cycle. When liver disease progresses, urea synthesis in the liver is reduced, leading to a reduction in the metabolism of ammonia, resulting in hyperammonemia $[33,34]$.

When blood ammonia levels are elevated as a result of a systemic "short circuit" or hepatic insufficiency, mitochondria disorder in astrocytes of the brain and changes in glutamate and glutamine occur, resulting in brain dysfunction $[35,36]$. Based on measurements of blood concentrations, MBDL surgery increased ammonia levels; notably, ammonia is an important factor in HE. Administration of $\mathrm{NaHS}$ restored ammonia levels. These findings suggest that NaHS may restore both liver function and ammonia levels.

MDA level, MPO activity, and CAT activity were measured to examine the antioxidative effects of NaHS. Changes in the proinflammatory cytokine TNF- $\alpha$ were also measured. CAT plays an important role in reducing free radicals and preventing oxidative stress in stagnant bile acids. In measurements of CAT activity, MBDL surgery reduced CAT levels, which caused oxidative stress $[37,38]$. Restoration of CAT levels by administration of $\mathrm{NaHS}$ indicated that NaHS reduced oxidative stress by MBDL and ameliorated liver dysfunction. MDA is the end-product of lipid peroxidation and is used as an antioxidant index. MBDL surgery increased MDA concentrations compared to those found in the sham group, and the effects of MBDL were reduced by NaHS administration. MPO is a marker of neutrophil infiltration and an index of both inflammation and oxidative stress. Administration of NaHS lowered MPO activity, which was increased by MBDL surgery. TNF- $\alpha$ plays an important role in liver damage caused by BDL $[39,40]$. In this study, administration of NaHS lowered the levels of TNF- $\alpha$ that had been increased following MBDL surgery. These findings indicate that NaHS decreased inflammatory responses. As a result, $\mathrm{NaHS}$ increased the activity of antioxidant enzymes and lessened neutrophil infiltration by reducing the release of inflammatory cytokines, such as TNF- $\alpha$.

NR2B is a subunit of the NMDA receptor that is important for learning and memory in the hippocampus. However, severe liver damage, such as BDL, rapidly increases levels of NMDA receptors and excessive NMDA receptor activity causes neurological disorders, such as cognitive and memory disorders [17]. $\mathrm{H}_{2} \mathrm{~S}$ may increase the production of cAMP and regulate the NMDA receptor [41]. In addition, $\mathrm{H}_{2} \mathrm{~S}$ attenuates NMDA-induced neuronal injury through its antioxidant effects [42]. When NR2B levels are decreased in $\mathrm{HIR}, \mathrm{H}_{2} \mathrm{~S}$ may increase the expression of NR2B and recover cognitive dysfunction [27]. Following MBDL surgery, the amount of NR2B in the hippocampus was increased compared to that observed in the sham group, indicating that NMDA receptor levels were increased. The increased amounts of NMDA receptors induced by MBDL surgery can cause neurotoxicity. The results of this experiment show that NaHS reduces the amount of NR2B that is induced by MBDL surgery. This may indicate that NaHS can reduce MBDL-induced neurotoxicity.

In conclusion, we found that NaHS reduces amounts of inflammatory cytokines and increases levels of antioxidants. In addition, these antioxidant and anti-inflammatory effects led to an amelioration of liver dysfunction. Recovery of liver function resulted in lower blood ammonia levels, leading to neurotoxic protective effects. These data support the potential for considering $\mathrm{NaHS}$ as a method for treating HE.

\section{ACKNOWLEDGEMENTS}

This study was supported by the Basic Science Research Program through the National Research Foundation of Korea and was funded by the Ministry of Education, Science and Technology (Grant 2016R1D1A1A09918019) and the Chung-Ang University Graduate Research Scholarship in 2018.

\section{CONFLICTS OF INTEREST}

The authors declare no conflicts of interest. 


\section{SUPPLEMENTARY MATERIALS}

Supplementary data including one figure can be found with this article online at http://pdf.medrang.co.kr/paper/pdf/Kjpp/ Kjpp2019-23-04-04-s001.pdf.

\section{REFERENCES}

1. Gow AG. Hepatic encephalopathy. Vet Clin North Am Small Anim Pract. 2017;47:585-599.

2. Gerber T, Schomerus H. Hepatic encephalopathy in liver cirrhosis: pathogenesis, diagnosis and management. Drugs. 2000;60:13531370.

3. Ong JP, Mullen KD. Hepatic encephalopathy. Eur J Gastroenterol Hepatol. 2001;13:325-334.

4. Mas A. Hepatic encephalopathy: from pathophysiology to treatment. Digestion. 2006;73 Suppl 1:86-93.

5. Ferenci P, Lockwood A, Mullen K, Tarter R, Weissenborn K, Blei AT. Hepatic encephalopathy--definition, nomenclature, diagnosis, and quantification: final report of the working party at the 11th World Congresses of Gastroenterology, Vienna, 1998. Hepatology. 2002;35:716-721.

6. Butterworth RF, Norenberg MD, Felipo V, Ferenci P, Albrecht J, Blei AT. Experimental models of hepatic encephalopathy: ISHEN guidelines. Liver Int. 2009;29:783-788.

7. Kountouras J, Billing BH, Scheuer PJ. Prolonged bile duct obstruction: a new experimental model for cirrhosis in the rat. Br J Exp Pathol. 1984;65:305-311.

8. Greve JW, Gouma DJ, Soeters PB, Buurman WA. Suppression of cellular immunity in obstructive jaundice is caused by endotoxins: a study with germ-free rats. Gastroenterology. 1990;98:478-485.

9. Rodrigo R, Jover R, Candela A, Compañ A, Sáez-Valero J, Erceg $\mathrm{S}$, Felipo V. Bile duct ligation plus hyperammonemia in rats reproduces the alterations in the modulation of soluble guanylate cyclase by nitric oxide in brain of cirrhotic patients. Neuroscience. 2005;130:435-443.

10. Huang YT, Hsu YC, Chen CJ, Liu CT, Wei YH. Oxidative-stressrelated changes in the livers of bile-duct-ligated rats. J Biomed Sci. 2003;10:170-178

11. Tag CG, Sauer-Lehnen S, Weiskirchen S, Borkham-Kamphorst E, Tolba RH, Tacke F, Weiskirchen R. Bile duct ligation in mice: induction of inflammatory liver injury and fibrosis by obstructive cholestasis. J Vis Exp. 2015;(96):52438.

12. Holecek M. Ammonia and amino acid profiles in liver cirrhosis: effects of variables leading to hepatic encephalopathy. Nutrition. 2015;31:14-20.

13. Giménez-Garzó C, Salhi D, Urios A, Ruíz-Sauri A, Carda C, Montoliu C, Felipo V. Rats with mild bile duct ligation show hepatic encephalopathy with cognitive and motor impairment in the absence of cirrhosis: effects of alcohol ingestion. Neurochem Res. 2015;40:230-240

14. Bliss TV, Collingridge GL. A synaptic model of memory: long-term potentiation in the hippocampus. Nature. 1993;361:31-39.

15. Felipo V. Hepatic encephalopathy: effects of liver failure on brain function. Nat Rev Neurosci. 2013;14:851-858.
16. Vieira MM, Schmidt J, Ferreira JS, She K, Oku S, Mele M, Santos AE, Duarte CB, Craig AM, Carvalho AL. Multiple domains in the C-terminus of NMDA receptor GluN2B subunit contribute to neuronal death following in vitro ischemia. Neurobiol Dis. 2016;89:223234.

17. Llansola M, Rodrigo R, Monfort P, Montoliu C, Kosenko E, Cauli O, Piedrafita B, El Mlili N, Felipo V. NMDA receptors in hyperammonemia and hepatic encephalopathy. Metab Brain Dis. 2007;22:321335.

18. Kim ST, Kyung EJ, Suh JS, Lee HS, Lee JH, Chae SI, Park ES, Chung YH, Bae J, Lee TJ, Lee WM, Sohn UD, Jeong JH. Phosphatidylcholine attenuated docetaxel-induced peripheral neurotoxicity in rats. Drug Chem Toxicol. 2018;41:476-485.

19. Wang D, Jacobs SA, Tsien JZ. Targeting the NMDA receptor subunit NR2B for treating or preventing age-related memory decline. Expert Opin Ther Targets. 2014;18:1121-1130.

20. Sun YR, Sun YG, Zhang Q, Wang XD, Wang X, Sun LJ. Learning and memory capacity and NMDA receptor expression in shen deficiency constitution rats. Zhongguo Zhong Xi Yi Jie He Za Zhi. 2016;36:597-601.

21. Bhambhani Y, Singh M. Physiological effects of hydrogen sulfide inhalation during exercise in healthy men. J Appl Physiol (1985). 1991;71:1872-1877.

22. Truong DH, Eghbal MA, Hindmarsh W, Roth SH, O'Brien PJ. Molecular mechanisms of hydrogen sulfide toxicity. Drug Metab Rev. 2006;38:733-744.

23. Hildebrandt TM. Modulation of sulfide oxidation and toxicity in rat mitochondria by dehydroascorbic acid. Biochim Biophys Acta. 2011;1807:1206-1213.

24. Calvert JW, Coetzee WA, Lefer DJ. Novel insights into hydrogen sulfide--mediated cytoprotection. Antioxid Redox Signal. 2010;12: 1203-1217.

25. Bos EM, Snijder PM, Jekel H, Weij M, Leemans JC, van Dijk MC, Hillebrands JL, Lisman T, van Goor H, Leuvenink HG. Beneficial effects of gaseous hydrogen sulfide in hepatic ischemia/reperfusion injury. Transpl Int. 2012;25:897-908.

26. Zhang Q, Fu H, Zhang H, Xu F, Zou Z, Liu M, Wang Q, Miao M, Shi X. Hydrogen sulfide preconditioning protects rat liver against ischemia/reperfusion injury by activating Akt-GSK-3 $\beta$ signaling and inhibiting mitochondrial permeability transition. PLoS One. 2013;8:e74422.

27. Tu FP, Li JX, Li Q, Wang J. Effects of hydrogen sulfide on cognitive dysfunction and NR2B in rats. J Surg Res. 2016;205:426-431.

28. Ahmadi S, Poureidi M, Rostamzadeh J. Hepatic encephalopathy induces site-specific changes in gene expression of GluN1 subunit of NMDA receptor in rat brain. Metab Brain Dis. 2015;30:1035-1041.

29. Singh S, Shackleton G, Ah-Sing E, Chakraborty J, Bailey ME. Antioxidant defenses in the bile duct-ligated rat. Gastroenterology. 1992;103:1625-1629.

30. Vázquez-Gil MJ, Mesonero MJ, Flores O, Criado M, Hidalgo F, Arévalo MA, Sánchez-Rodríguez A, Tuñón MJ, López-Novoa JM, Esteller A. Sequential changes in redox status and nitric oxide synthases expression in the liver after bile duct ligation. Life Sci. 2004;75:717-732.

31. Nam Y, Bae J, Jeong JH, Ko SK, Sohn UD. Protective effect of ultrasonication-processed ginseng berry extract on the $\mathrm{D}$-galactosamine/lipopolysaccharide-induced liver injury model in rats. $J$ 
Ginseng Res. 2018;42:540-548.

32. Song Z, Deaciuc I, Song M, Lee DY, Liu Y, Ji X, McClain C. Silymarin protects against acute ethanol-induced hepatotoxicity in mice. Alcohol Clin Exp Res. 2006;30:407-413.

33. Katayama K. Ammonia metabolism and hepatic encephalopathy. Hepatol Res. 2004;30S:73-80.

34. Lockwood AH. Blood ammonia levels and hepatic encephalopathy. Metab Brain Dis. 2004;19:345-349.

35. Butterworth RF, Giguère JF, Michaud J, Lavoie J, Layrargues GP. Ammonia: key factor in the pathogenesis of hepatic encephalopathy. Neurochem Pathol. 1987;6:1-12.

36. Norenberg MD. Astrocytic-ammonia interactions in hepatic encephalopathy. Semin Liver Dis. 1996;16:245-253.

37. Jang HS, Um SI, Lee SH, Whang WK, Min YS, Park SY, Sohn UD. The protective mechanism of QGC in feline esophageal epithelial cells by interleukin-1 $\beta$ treatment. Arch Pharm Res. 2017;40:204-213.

38. Lee YJ, Kim SJ, Kwon KW, Lee WM, Im WJ, Sohn UD. Inhibitory effect of FSLLRY-NH $\mathrm{N}_{2}$ on inflammatory responses induced by hydrogen peroxide in HepG2 cells. Arch Pharm Res. 2017;40:854-863.

39. Plebani M, Panozzo MP, Basso D, De Paoli M, Biasin R, Infantolino D. Cytokines and the progression of liver damage in experimental bile duct ligation. Clin Exp Pharmacol Physiol. 1999;26:358-363.

40. Fernández-Martínez E, Pérez-Alvarez V, Tsutsumi V, Shibayama M, Muriel P. Chronic bile duct obstruction induces changes in plasma and hepatic levels of cytokines and nitric oxide in the rat. Exp Toxicol Pathol. 2006;58:49-58.

41. Kimura H. Hydrogen sulfide induces cyclic AMP and modulates the NMDA receptor. Biochem Biophys Res Commun. 2000;267:129133.

42. Sakamoto K, Suzuki Y, Kurauchi Y, Mori A, Nakahara T, Ishii K. Hydrogen sulfide attenuates NMDA-induced neuronal injury via its anti-oxidative activity in the rat retina. Exp Eye Res. 2014;120:9096. 


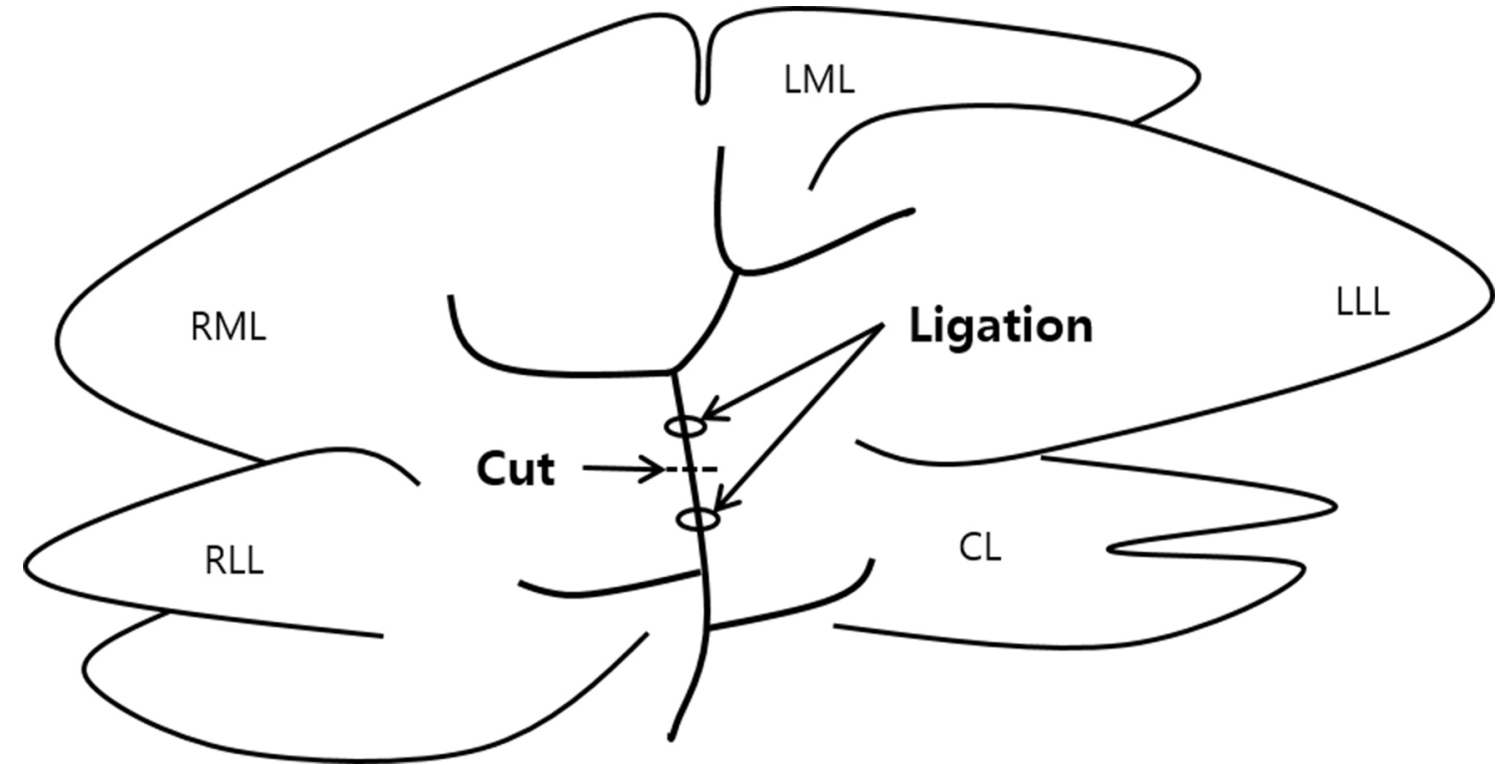

Supplementary Fig. 1. Schematic of mild bile duct ligation in rat. Rat biliary ducts consists of five branch. The middle of the bild duct was ligated and cutted to ligate only upper three bile ducts (RLL, right lateral lobe; RML, right medial lobe; LML, left medial lobe; LLL, left lateral lobe; CL, caudate lobe). 